\title{
Early Postoperative Aortic Root Thrombus After Heartmate 3
}

\author{
Jama Jahanyar, MD, PhD,${ }^{1}$ Joshua M. Liao, MD, MSc, ${ }^{2}$ Patrick DeValeria, MD, ${ }^{1}$ D. Eric Steidley, MD, \\ Ryan Craner, MD, ${ }^{4}$ Harish Ramakrishna, $\mathrm{MD},{ }^{4}$ Robert L. Scott, $\mathrm{MD}, \mathrm{PhD}^{3}$ \\ ${ }^{1}$ Department of Cardiothoracic Surgery, Mayo Clinic Hospital, Scottsdale/Phoenix, AZ; ${ }^{2}$ Department of Medicine, University \\ of Washington, Seattle, WA; ${ }^{3}$ Division of Cardiology, Department of Medicine, Mayo Clinic Hospital, Scottsdale/Phoenix, AZ; \\ ${ }^{4}$ Department of Anesthesiology, Mayo Clinic Hospital, Scottsdale/Phoenix, AZ
}

\section{ABSTRACT}

As a bridge to heart transplantation or destination treatment, implantation of the Heartmate 3 (HM3) left ventricular assist device is a viable option for patients with end-stage congestive heart failure. The recent Momentum 3 trial has shown favorable outcomes compared with Heartmate 2. We report the first case of aortic root thrombus occurring early after HM3 implantation as a bridge to heart transplantation. Our case suggests that bridging with an Impella 5.0 preceding HM3 implantation could potentially predispose patients to aortic root thrombus after HM3 implantation, due to Impella-related injury to the aortic valve and aortic root stasis after durable LVAD support.

\section{INTRODUCTION}

Due to the scarcity of heart allografts, patients with endstage congestive heart failure frequently require temporary therapies as a safe bridge to heart transplantation. Options range from chemical (e.g. inotropes and inodilators) [Assad-Kottner 2008] to mechanical (e.g. IABP, ECMO, LVAD) therapies, with durable continuous flow LVADs, such as the Heartmate 3 (HM3) representing particularly promising options with good midterm results. For example, the recent Momentum 3 trial demonstrated that the HM3 LVAD had favorable results compared with its predecessor, the Heartmate 2 [Mehra 2019]. Another increasingly important strategy for treating patients with congestive heart failure and acute cardiogenic shock involves bridging to durable LVAD using a temporary LVAD, such as the Impella 5.0 [Lima 2016], a microaxial pump that can deliver up to $5.0 \mathrm{~L} / \mathrm{min}$ of forward flow. This helps bridge patients by reversing end-organ failure and deconditioning, thereby improving results after durable LVAD implantation or heart transplantation.

Received fune 26, 2019; accepted fuly 24, 2019.

Correspondence: Jama Jahanyar, MD, PbD, FACS, Assistant Professor of Surgery, Dept. of Cardiothoracic Surgery, Mayo Clinic, 5777 East Mayo Boulevard, Phoenix, AZ 85054; (e-mail: jjahanyar@gmail.com).

\section{CASE REPORT}

A 64-year-old man with ischemic cardiomyopathy, acute cardiogenic shock, and end-organ dysfunction required urgent implantation of an Impella 5.0 LVAD via the right axillary artery. The Impella was able to stabilize the patient and reverse end-organ dysfunction, with the patient significantly improving and becoming ambulatory while on this support. This improvement led to an initial decision to wait on durable LVAD implantation and instead attempt to bridge the patient directly to heart transplantation.

Unfortunately, a donor organ could not be allocated to the patient during 5 weeks of Impella 5.0 LVAD support. Therefore, the decision was made to proceed with a more durable LVAD using the HM3 as a bridge to heart transplantation. The HM3 implantation and early postoperative course was unremarkable. The LVAD was well positioned, with an angled and beveled outflow graft to the ascending aorta (Figure 1). Anticoagulation with Coumadin and Aspirin was started within 12 hours after implantation. No heparin bridge was used, which is our standard protocol. The INR was therapeutic on postoperative Day 2. However, on postoperative Day 4, a routine echocardiogram demonstrated a large aortic root thrombus in all three cusps of the aortic valve (Figure 2). Because the patient was doing well clinically, treatment with systemic anticoagulation initially was attempted. However, serial imaging did not reveal reductions in thrombus size over 3 days and given concerns for thrombus propagation into the coronary ostia and acute right heart failure or fatal stroke, the patient urgently was listed for heart transplantation under special exemption. He successfully was transplanted 9 days after HM3 implantation, with an uneventful subsequent course over the following 6 months.

\section{DISCUSSION}

To our knowledge, this is the first case of an early acute aortic root thrombus after HM3 LVAD implantation. Aortic root thrombus formation after continuous flow LVAD has been described [Fried 2018], but not after HM3 or in the setting of Impella bridge to durable LVAD. Ultimately, the leading suspicion was that the Impella caused secondary aortic valve changes that served as a potential nidus for clot formation, the likelihood of which was increased in the setting of the HM3 (i.e., unloaded heart with a closed aortic valve and aortic root stasis). This possibility was supported by 


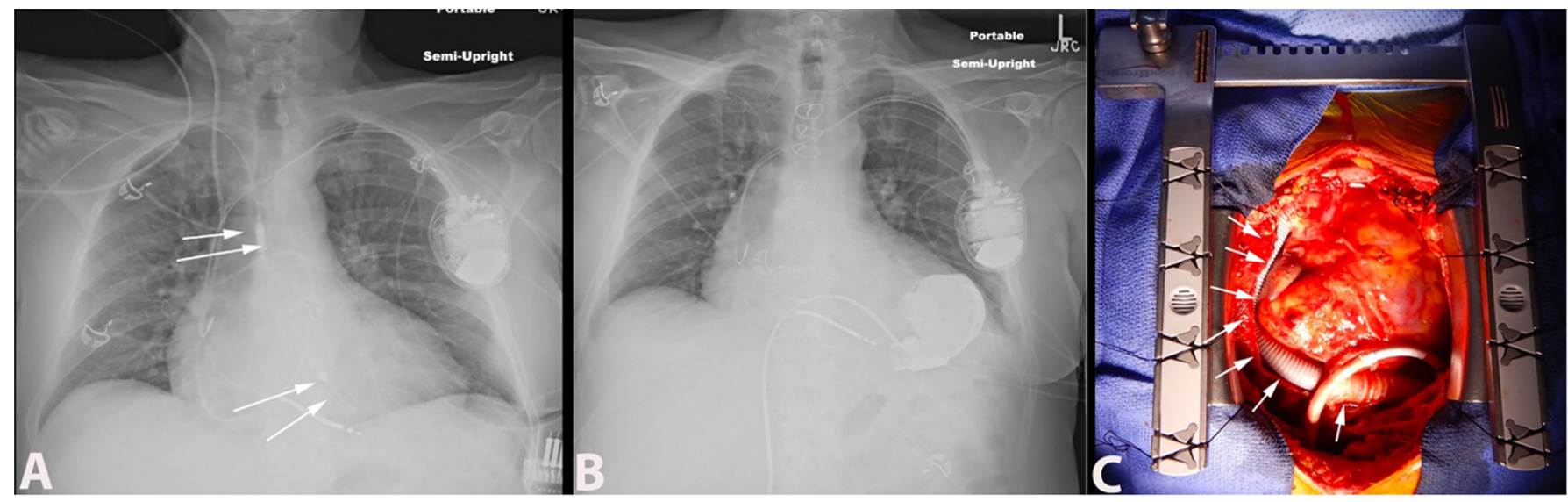

Figure 1. A) CXR; Impella as bridge to durable LVAD. Inflow and outflow well positioned in LV and ascending aorta, respectively. B) CXR; Well positioned HM3 pump. C) Intraoperative picture; Well aligned, angled and beveled outflow graft to the ascending aorta.

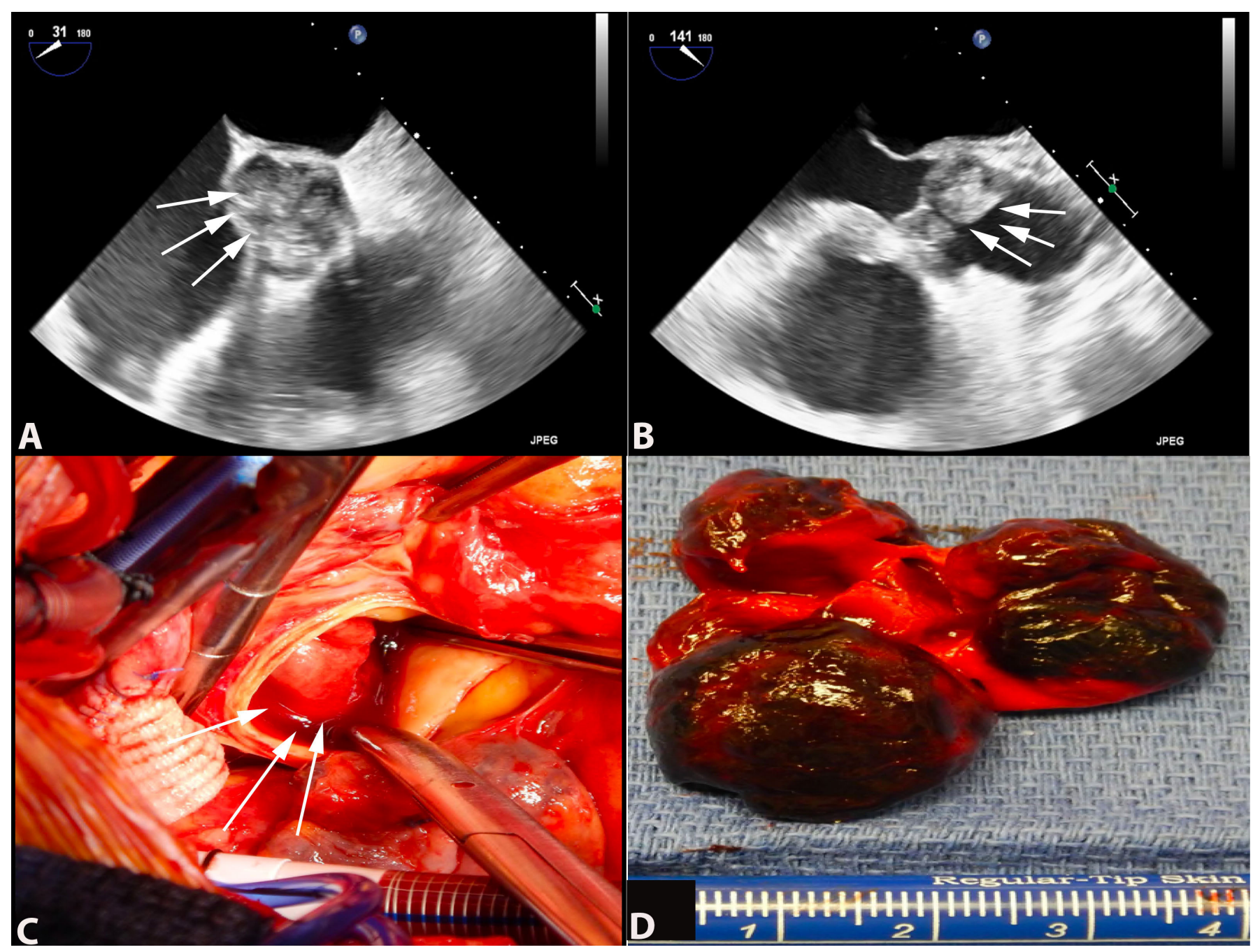

Figure 2. A) TEE short axis; large aortic root thrombus in all 3 aortic cusps. B) TEE long axis; large aortic root thrombus. C) Intraoperative picture; large thrombus in the aortic root. D) Large organizing laminated thrombus. 
pathologic evaluation of the aortic valve, which demonstrated localized trauma to the right coronary leaflet with evidence of non-infectious endocarditis.

Although aortic root thrombus has not yet been described after HM3 support, incidents are likely to emerge as experience with HM3 increases. Despite favorable reports from Momentum 3, aortic root thrombus is a known complication of continuous flow LVADs, now also including the HM3.

While more data are needed to delineate the exact mechanisms of aortic root thrombus formation early after HM3 implantation, our case demonstrates that secondary aortic valve abnormalities represents one possible risk factor. Vigilance about risk factors and thrombus complications are critical, particularly given the potential for severe consequences (e.g., acute right heart failure, fatal stroke) of thrombus propagation.

Importantly, our case highlights the complexity of managing patients with acute cardiogenic shock requiring definitive therapy. In the case of durable LVAD, the HM3 pump is able to completely unload the left ventricle, thereby rendering the aortic valve completely closed for prolonged periods of time. In patients with injured aortic valves, such dynamics may have serious consequences. While the issue could potentially be prevented by alternatives that achieve intermittent aortic valve opening rather than completely unloading the heart, they may not provide the support needed to reverse endorgan failure. Regardless, these are important considerations given growing utilization of temporary LVADs as bridges to more durable LVADs, and should be factored into clinical decision-making, and possibly, the choice of durable LVAD.

\section{REFERENCES}

Assad-Kottner C, Chen D, Jahanyar J, et al. 2008. The use of continuous milrinone therapy as bridge to transplant is safe in patients with short waiting times. J Card Fail 14 (10): 839-43.

Fried J, Garan AR, Shames S, et al. 2018. Aortic root thrombosis in patients supported with continuous-flow left ventricular assist devices. J Heart Lung Transplant 37 (12): 1425-1432.

Lima B, Kale P, Gonzalez-Stawinski GV, Kuiper JJ, Carey S, Hall SA. 2016. Effectiveness and Safety of the Impella 5.0 as a Bridge to Cardiac Transplantation or Durable Left Ventricular Assist Device. Am J Cardiol 117 (10): 1622-1628.

Mehra MR, Uriel N, Naka Y, et al. 2019. A Fully Magnetically Levitated Left Ventricular Assist Device - Final Report. N Engl J Med 380 (17): 1618-1627. 